\title{
Fluctuation spectroscopy of granularity in superconducting structures
}

\author{
I. V. Lerner, ${ }^{1,2}$ A. A. Varlamov, ${ }^{3,2}$ and V. M. Vinokur ${ }^{2}$ \\ ${ }^{1}$ School of Physics and Astronomy, University of Birmingham, Edgbaston, B15 2TT Birmingham, UK \\ ${ }^{2}$ Materials Science Division, Argonne National Laboratory, 9700 S.Cass Avenue, Argonne Il 60439 \\ ${ }^{3}$ COHERENTIA-INFM, CNR, Viale del Politecnico 1, I-00133 Rome, Italy
}

(Dated: submitted 16 July 2007; published 21 March 2008)

\begin{abstract}
We suggest to use 'fluctuation spectroscopy' as a method to detect granularity in a disordered metal close to a superconducting transition. We show that with lowering temperature $T$ the resistance $R(T)$ of a system of relatively large grains initially grows due to the fluctuation suppression of the one-electron tunneling but decreases with further lowering $T$ due to the coherent charge transfer of the fluctuation Cooper pairs. Under certain conditions, such a maximum in $R(T)$ turns out to be sensitive to weak magnetic fields due to a novel Maki - Thompson type mechanism.

PACS numbers: $74.81 . \mathrm{Bd}, 72.15 .-\mathrm{v}, 73.23 .-\mathrm{b}$
\end{abstract}

Since seminal experiments on the superconductinginsulator transition in granular samples [1], transport properties of granular metals enjoy extensive attention, see [2, 3] for reviews and references. Granularity offers systems with tuneable parameters eminently suitable for studies of the interplay between electron correlations and mesoscopic effects of disorder. Generically, effects of granularity are most profound at $T \gtrsim \Gamma$ ( $\Gamma$ is the electron tunneling rate between the grains). At low temperatures $(T \lesssim \Gamma)$ transport properties of the granular metal coincide with those of amorphous one [4] including rather subtle effects of weak localization [5, $[6]$. The role of granularity is widely believed to be crucial for the metal-insulator and superconductor-insulator transitions. It was recently discovered [7, 8] that it may be of self-induced nature and appear even in homogeneously disordered samples. This poses an interesting question whether it is possible to find a reliable experimental benchmark of granularity in electronic transport in the metallic regime.

In this Letter we show that there exists a firm experimental signature of granularity - the appearance of a maximum in the temperature dependence of the resistance $R(T)$, provided that a granular system is made of material which experiences the superconducting transition. The magnitude and position of such a maximum might be very sensitive to a weak magnetic field. The maximum is due to an interplay of different types of superconducting fluctuation contributions, specific for granular systems, at temperatures $T \gtrsim T_{\mathrm{c}}$ (the transition temperature). All this is strikingly different from a monotonic decrease with $T$ of the fluctuation resistance of amorphous systems close to $T_{\mathrm{c}}$ and allows one to extract various characteristics of the granularity, thus suggesting a method of characterization of disordered systems which can be termed as the fluctuation spectroscopy.

The disordered system becomes effectively granular for one-electron transport provided that the tunneling conductance between inhomogeneities (grains), $g_{\mathrm{T}} \sim \Gamma / \delta$, is much smaller than the intragranular conductance $g \sim$
$E_{\mathrm{Th}} / \delta$ - in the opposite case, the system is indistinguishable from the amorphous one. Furthermore, the granular character is preserved also for the charge transfer by fluctuation Cooper pairs when their Ginzburg - Landau life-time, $\tau_{\mathrm{GL}} \sim\left(T-T_{\mathrm{c}}\right)^{-1}$ is much shorter than the escape time $\Gamma^{-1}$ (we use units with $\hbar=k_{\mathrm{B}}=1$ ). Finally, the system remains metallic when $g_{\mathrm{T}} \gg 1$. The above conditions can be satisfied when

$$
\delta \lesssim \Gamma \lesssim E_{\mathrm{Th}}, T_{\mathrm{c}}
$$

Here $\delta$ is the mean level spacing in the grain, $E_{\mathrm{Th}}=\mathcal{D} / d^{2}$ is its Thouless energy, $d$ is the typical grain size, and $\mathcal{D}$ is the intragrain diffusion coefficient [9]. We will focus at the case $E_{\mathrm{Th}} \lesssim T_{\mathrm{c}}$ where the Cooper pair intragrain motion can have both the three-dimensional $(3 D)$ and zero-dimensional $(0 D)$ character.

The characteristic feature of the fluctuation pairing in the problem is the appearance of two different scales for the superconducting coherence length, $\xi_{\mathrm{g}}=\left(\mathcal{D} / T_{\mathrm{c}}\right)^{1 / 2}$ and $\xi_{\mathrm{T}}=\left(\Gamma d^{2} / T_{\mathrm{c}}\right)^{1 / 2}$, driven by the intragrain and intergrain pairing, respectively. The above inequalities correspond to the length scales ranging as $\xi_{\mathrm{T}} \lesssim \xi_{\mathrm{g}} \lesssim d$.

The existence of the two correlation scales becomes crucial in the vicinity of $T_{\mathrm{c}}$ where the superconducting fluctuations are governed by the temperature-dependent Ginzburg-Landau correlation length $\xi(\epsilon)$ :

$$
\xi_{\mathrm{g}}(\epsilon) \equiv \frac{\xi_{\mathrm{g}}}{\sqrt{\epsilon}}, \quad \xi_{\mathrm{T}}(\epsilon) \equiv \frac{\xi_{\mathrm{T}}}{\sqrt{\epsilon}}, \quad \epsilon \equiv \frac{T-T_{\mathrm{c}}}{T_{\mathrm{c}}} .
$$

This leads to the existence of three distinct temperature regimes near $T_{\mathrm{c}}$ (see Fig. 1):

$$
\begin{aligned}
\epsilon_{\mathrm{g}} \lesssim \epsilon & \lesssim 1, \\
\epsilon_{\mathrm{T}} \lesssim \epsilon & \lesssim \epsilon_{\mathrm{g}}, \\
\epsilon & \lesssim \epsilon_{\mathrm{T}},
\end{aligned}
$$

where $\epsilon_{\mathrm{T}} \equiv \Gamma / T_{\mathrm{c}}$ and $\epsilon_{\mathrm{g}} \equiv E_{\mathrm{Th}} / T_{\mathrm{c}}$. For the first two regimes, it is the intragrain correlations which govern the 


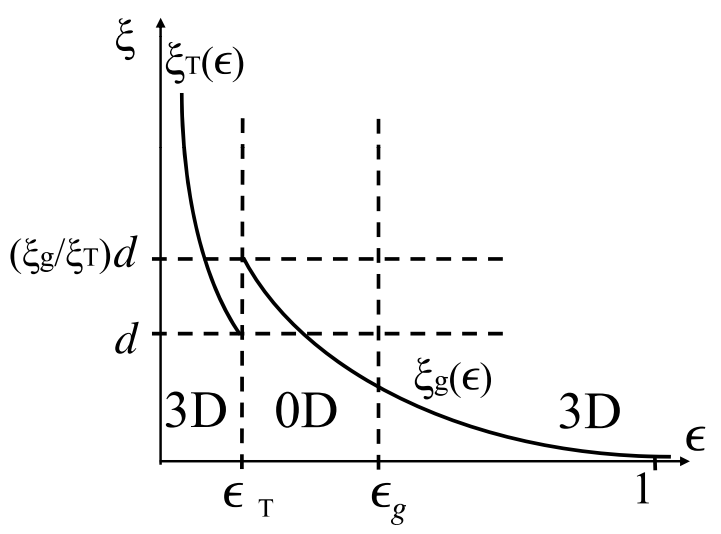

FIG. 1: Temperature dependence of the Ginzburg-Landau superconducting coherence length above $T_{\mathrm{c}}$ : at $\epsilon=\epsilon_{\mathrm{T}}$ it jumps from the intra- to inter-granular regime.

fluctuation corrections to the conductance, these correlations having a $3 D$ character in region (2), albeit very different from that in the amorphous metal, and a $0 D$ character in region (3). For smaller grains, when $E_{\mathrm{Th}} \gtrsim T_{\mathrm{c}}$, region (2) vanishes which does not change the qualitative picture. In the immediate vicinity of $T_{\mathrm{c}}$, regime (4), the intergrain fluctuation pairing dominates. In this case, the fluctuation corrections are equivalent to those in amorphous disordered media with the effective diffusion coefficient $D_{\mathrm{T}}=\Gamma d^{2}$. It is the existence of the regimes (2) and (3) which imprints a pronounced signature of the granularity as we now demonstrate.

The model under consideration is defined by the standard Hamiltonian,

$$
\hat{H}=\hat{H}_{0}+\hat{H}_{\mathrm{T}} .
$$

where $\hat{H}_{0}$ describes electrons in a single grain in the presence of the BCS pairing (characterized by the transition temperature $T_{\mathrm{c}}$ ) and disorder (characterized by the intragrain diffusion coefficient $\mathcal{D}$ ), while $\hat{H}_{\mathrm{T}}$ describes the intergrain tunneling with the amplitude $t$ which is assumed to be local (i.e. momentum-independent) and the same for all the grain pairs. For simplicity, the grains are assumed to have a spherical shape of diameter $d[10]$.

The tunneling current between the neighboring grains can be expressed as follows [11]:

$$
I(V)=-\left.e \operatorname{Im} K^{\mathrm{R}}(\omega)\right|_{\omega=-\mathrm{i} e V},
$$

where $K^{\mathrm{R}}(\omega)$ is the analytical continuation, $\mathrm{i} \omega_{\nu} \rightarrow \omega$, to the upper half-plane of the current-current correlation function in the Matsubara frequencies

$$
\mathcal{K}\left(\omega_{\nu}\right)=T|t|^{2} \sum_{\varepsilon_{n}} \sum_{\boldsymbol{p}, \boldsymbol{p}^{\prime}} \mathcal{G}_{L}\left(\boldsymbol{p}, \varepsilon_{n}+\omega_{\nu}\right) \mathcal{G}_{R}\left(\boldsymbol{p}^{\prime}, \varepsilon_{n}\right) .
$$

Here $\mathcal{G}_{L, R}$ are the exact electron Green's functions in the neighboring ("left" and "right") grains with $\varepsilon_{n}=$ $\pi T(2 n+1)$ and $\omega_{m}=2 \pi T m$ being the fermionic and bosonic Matsubara frequencies. Hence the conductivity

$$
\sigma_{\mathrm{T}}=\frac{1}{d} \frac{\mathrm{d} I}{\mathrm{~d} V}=\left.\frac{e^{2}}{d} \operatorname{Im} \frac{\mathrm{d} K^{R}(\omega)}{\mathrm{d} \omega}\right|_{\omega \rightarrow 0},
$$

where $V$ is the voltage drop at the grain.

Examples of diagrams describing significant fluctuation contributions to $\mathcal{K}\left(\omega_{m}\right)$ are presented in Fig. 2. The solid lines correspond to the disorder-averaged electron Green's functions, the circled crosses represent the tunneling amplitude $t$, and the wavy lines correspond to the fluctuation propagator, $\mathcal{L}\left(q_{k}, \Omega_{n}\right)$. For a single superconducting grain $\mathcal{L}$ is found by solving the linearized Ginzburg-Landau equation with the boundary condition corresponding to the zero current flow at the grain surface. It has the standard form [12] at $\epsilon \ll 1$ but with quantized momenta $q_{k}$. In what follows we will need the analytic continuation of this propagator to the upper half-plane given by

$$
L^{\mathrm{R}}\left(q_{k}, \Omega\right)=-\frac{1}{\nu} \frac{1}{\epsilon-i \pi \Omega / 8 T_{\mathrm{c}}+\xi_{\mathrm{g}}^{2} q_{k}^{2}} .
$$

Here $\nu \sim 1 / d^{3} \delta$ is the density of states (DoS), and the quantized momenta are defined by $q_{k} d=2 \tan \left(q_{k} d / 2\right)$ which gives $q_{0}=0$ and $q_{k} d \approx 2 \pi k+\pi$ for $k \geq 1$. Finally, the shaded triangles in Fig. 2 are the Cooperons describing the usual 'dressing' of the fluctuation propagator due to the coherent electron scattering from impurities,

$$
\lambda\left(q_{k}, \varepsilon_{1}, \varepsilon_{2}\right)=\frac{\tau^{-1}}{\left|\varepsilon_{1}-\varepsilon_{2}\right|+\mathcal{D} q_{k}^{2}} .
$$

Let us first consider the immediate vicinity of $T_{\mathrm{c}}$, region (4) where the intergrain coherence length $\xi_{\mathrm{T}}(\epsilon)$ exceeds the grain size. Then that the granularity is practically irrelevant and the system behaves as the effective amorphous metal with the diffusion coefficient $D_{\mathrm{T}}$. In this region the fluctuation correction $\sigma$ is dominated by the Aslamazov - Larkin (AL) diagram, Fig. 22r, and increases in accordance with the standard $3 D$ result [12]:

$$
\sigma_{A L}^{(\mathrm{eff})}(\epsilon) \sim \frac{e^{2}}{\xi_{\mathrm{T}}} \frac{1}{\sqrt{\epsilon}} .
$$

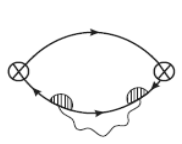

a

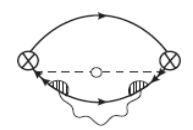

b

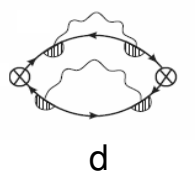

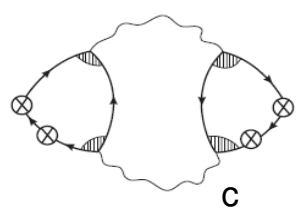

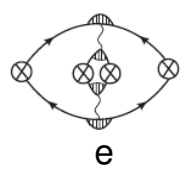

FIG. 2: Fluctuation corrections to the tunneling current in the lowest orders in transparency and fluctuations. 
On the contrary, in regions (2) and (3) the granularity is paramount. We will show that this may result in the appearance of a maximum in the temperature dependence of resistance. We start our consideration for this region with the first order fluctuation contribution to $\sigma$, Eq. (5), corresponding to the DoS fluctuating separately in each of the grains (two such contributions are shown in Fig. 2a,b). At $\epsilon \ll 1$ the fluctuation propagator, Eq. (6) ), should be taken at $\Omega=0$. Integrating over the electron momenta and summing over the fermionic frequencies one finds the following negative contribution:

$$
\sigma_{\mathrm{DoS}}(\epsilon) \sim-\frac{e^{2}}{d} \epsilon_{\mathrm{T}} \sum_{k} \frac{1}{\epsilon+\xi_{\mathrm{g}}^{2} q_{k}^{2}} .
$$

In region (2), i.e. for the intragranular $3 D$ motion, the summation in Eq. (11) is reduced to integration, while in the $0 D$ region (3) only the $q_{0}=0$ term in Eq. (9) contributes which gives

$$
\sigma_{\operatorname{DoS}}(\epsilon) \sim-\frac{e^{2}}{d} \epsilon_{\mathrm{T}} \times\left\{\begin{array}{cc}
\frac{1}{\sqrt{\epsilon_{\mathrm{g}} \epsilon}}, & \epsilon_{\mathrm{g}} \lesssim \epsilon \lesssim 1 \\
\frac{1}{\epsilon}, & \epsilon_{\mathrm{T}} \lesssim \epsilon \lesssim \epsilon_{\mathrm{g}} .
\end{array} .\right.
$$

The AL contribution to the correlation function of Eq. (5), diagram 25. [13], is of the second order in $\epsilon_{\mathrm{T}} \equiv$ $\Gamma / T_{\mathrm{c}}$, but it is more singular in $1 / \epsilon$ than the first-order DoS one above. It is given by

$$
\mathcal{K}_{\mathrm{AL}}\left(\omega_{\nu}\right)=T \sum_{\Omega_{n}} \sum_{k, l}\left|B_{k l}\right|^{2} \mathcal{L}\left(q_{k}, \Omega_{n}+\omega_{\nu}\right) \mathcal{L}\left(q_{l}, \Omega_{n}\right) .
$$

Here $B_{k l}$ denotes the loop made of four Green's functions (Fig. 22 c) with the Cooperon dressing (77). The standard calculation [12] gives $B_{k l} \sim \nu \epsilon_{\mathrm{T}}$. Now one transforms the sum over $\Omega_{n}$ into a contour integral in a usual way, making appropriate cuts in the complex plane of the frequency $z$ and thus constructing the analytical continuation $\mathrm{i} \omega_{\nu} \rightarrow \omega+\mathrm{i} 0$ which yields in the limit $\omega \rightarrow 0$ the following expression for the AL contribution into the fluctuation conductivity:

$$
\begin{aligned}
\sigma_{\mathrm{AL}}(\epsilon) & \sim \frac{e^{2}}{d} \epsilon_{\mathrm{T}}^{2} \int_{-\infty}^{\infty} \mathrm{d} \zeta\left(\sum_{k} \frac{1}{\left(\epsilon+\xi_{\mathrm{g}}^{2} q_{k}^{2}\right)^{2}+\zeta^{2}}\right)^{2} \\
& \sim \frac{e^{2}}{d} \epsilon_{\mathrm{T}}^{2} \times\left\{\begin{array}{l}
\frac{1}{\epsilon_{\mathrm{g}} \epsilon^{2}}, \epsilon_{\mathrm{g}} \lesssim \epsilon \lesssim 1 \\
\frac{1}{\epsilon^{3}}, \quad \epsilon_{\mathrm{T}} \lesssim \epsilon \lesssim \epsilon_{\mathrm{g}}
\end{array}\right.
\end{aligned}
$$

Two types of the Maki - Thompson (MT) contribution are represented by diagrams in Fig. 2 d,e. On the face of it, diagram $2 \mathrm{~d}$ is just a second order contribution from the DoS fluctuations. But this is not so. The phase coherence of the fluctuation propagators in two grains is absolutely essential here so that one may classify this diagram as belonging to the Maki - Thompson type. Its leading contribution describes the interference between the DoS fluctuations in the neighboring grains while only the sub-leading one contributes to the second order DoS corrections. Technically, it results from the summation over the anomalous interval where the fermionic frequencies in the Cooperons (shaded triangles, each given by Eq. (7)) are of the opposite sign. This results in the appearance, alongside with the two fluctuation propagators, of an additional strongly singular factor, cut off by the intragrain dephasing rate $\gamma_{\phi}=1 /\left(T_{\mathrm{c}} \tau_{\phi}\right)$ :

$$
\sigma_{\mathrm{MT}} \sim \frac{e^{2}}{d} \frac{\epsilon_{\mathrm{T}}^{2}}{g_{\mathrm{T}}} \sum_{k, l} \frac{1}{\epsilon+\xi_{\mathrm{g}}^{2} q_{k}^{2}} \frac{1}{\epsilon+\xi_{\mathrm{g}}^{2} q_{l}^{2}} \frac{1}{\left[2 \gamma_{\varphi}+\xi_{\mathrm{g}}^{2} q_{k}^{2}+\xi_{\mathrm{g}}^{2} q_{l}^{2}\right]^{3}} .
$$

Although this contribution is of the first order in $\Gamma$, its overall factor $\epsilon_{\mathrm{T}}^{2} / g_{\mathrm{T}}$ is smaller than that in the AL contribution, which is of the second order in $\Gamma$. The extra factor $1 / g_{\mathrm{T}} \equiv \delta / \Gamma$ is due to the reduction of the effective phase volume in the former contribution which contains less independent integrations over fast electronic momenta. A straightforward estimation of the above summation gives

$$
\sigma_{\mathrm{MT}}(\epsilon) \sim \frac{e^{2}}{d} \frac{\epsilon_{\mathrm{T}}^{2}}{g_{\mathrm{T}} \gamma_{\varphi}^{3}} \times \begin{cases}\frac{\gamma_{\varphi}}{\epsilon_{\mathrm{g}} \epsilon^{2}}, & \epsilon_{\mathrm{g}} \lesssim \gamma_{\varphi} \lesssim \epsilon \lesssim 1 \\ \frac{1}{\epsilon_{\mathrm{g}} \epsilon}, & \epsilon_{\mathrm{g}} \lesssim \epsilon \lesssim \gamma_{\varphi} \lesssim 1 \\ \frac{1}{\epsilon^{2}}, & \epsilon_{\mathrm{T}} \lesssim \min \left\{\epsilon, \gamma_{\phi}\right\} \lesssim \epsilon_{\mathrm{g}}\end{cases}
$$

The magnitude of this contribution differs from the AL one, Eq. (11), by the factor $g_{\mathrm{T}} \gamma_{\phi}^{3}$, which may be either small or large. The dephasing rate $\gamma_{\phi}$ is the sum of the escape rate, $\gamma_{\phi}^{\text {esc }} \sim \Gamma / T_{\mathrm{c}} \equiv \epsilon_{\mathrm{T}}$, and the dephasing rate due to the electron-electron interaction, $\gamma_{\phi}^{e e}=\epsilon_{\mathrm{T}} /\left(g_{\mathrm{T}} \epsilon_{g}^{\alpha}\right)$ with $\alpha=3 / 2$ in region (2) and $\alpha=2$ in region (3) [14].

The contribution of diagram 2 , which is a modification of the standard MT diagram for the present case, is smaller by the factor $\epsilon_{\mathrm{T}}$ than the expression in Eq. (12).

Let us discuss the results obtained. On the face of it, Eq. (11) contradicts to the well known results for the conductivity [12], $\sigma_{A L}^{(D)} \sim \epsilon^{\frac{D}{2}-2}$, which are supposed to be applicable to any dimensionality $D$. This apparent discrepancy is due to the tunneling character of the fluctuation Cooper pair motion between grains. The Cooper pair tunneling at $T>T_{\mathrm{c}}$ requires two independent electron hops, the probability of each being proportional to the tunneling rate $\Gamma$. To preserve the superconducting coherence both should occur within the Cooper pair lifetime $\tau_{\mathrm{GL}} \sim 1 / \epsilon T_{\mathrm{c}}$. Thus the probability $W$ of the pair tunneling between two grains $W=\Gamma^{2} \tau_{\mathrm{GL}} \propto 1 / \epsilon$ acts as the correction factor to the expected $0 D$ expression $\propto \epsilon^{-2}$ which leads us to the result (11). In the same spirit one can obtain qualitatively the $3 D$ asymptotic of Eq. (11), valid when $\xi_{g} \ll d$. The fluctuation conductivity in such a $3 D$ grain is the standard $\sigma_{\mathrm{AL}}^{(3)} \sim \epsilon^{-1 / 2}$. Then the probability $W$ above is reduced due to the fact that 


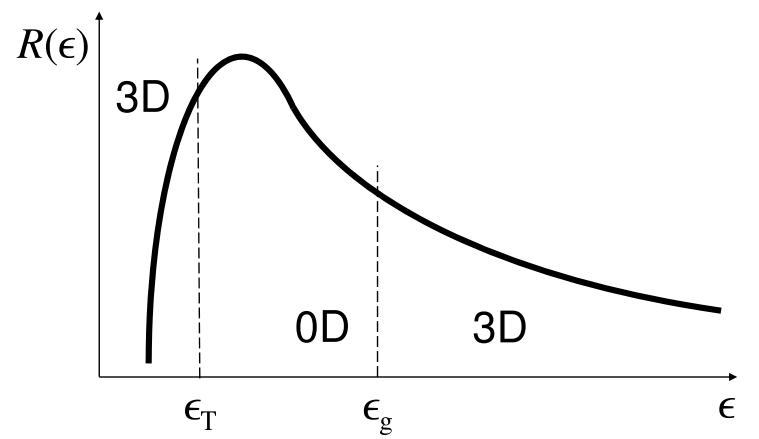

FIG. 3: Temperature dependence of the granular metal resistance in the vicinity of superconducting transition for $\epsilon_{\mathrm{g}}^{2} \lesssim \epsilon_{\mathrm{T}}$.

only the pairs in the skin layer of thickness $\xi_{\mathrm{g}}(\epsilon)$ near the grain boundary can tunnel, their fraction in the grain being $\xi_{\mathrm{g}}(\epsilon) / d$. This immediately leads to the appropriate asymptotics of Eq. (11).

Finally let us discuss the temperature dependence of the fluctuation contribution to the conductivity given by the sum of Eqs. (10)-(12). For $\gamma_{\phi}^{3} g_{\mathrm{T}} \gg 1$, it is determined by the competition of the negative $\sigma_{\mathrm{DoS}}$ and positive $\sigma_{\mathrm{AL}}$. At the onset of the fluctuation region, $\epsilon \lesssim 1$, the former dominates since it is proportional to the lowest power of the small tunneling parameter $\epsilon_{\mathrm{T}}$ resulting in the initial increase of the resistance with decrease of $\epsilon \equiv T / T_{\mathrm{c}}-1$. With further decrease of $\epsilon$, the AL contribution, more singular in $\epsilon^{-1}$ inevitably wins. The resulting maximum in the temperature dependence of resistance $R(\epsilon)$ occurs in region (2) for $\epsilon_{\mathrm{g}}^{2}<\epsilon_{\mathrm{T}}$, or in region (3) in the opposite case - the latter is illustrated in Fig. 3.

In the case of weak dephasing, the MT contribution, Eq. (12), takes over the AL one, Eq. (11). If dephasing is so small that $\gamma_{\phi}^{3} g_{\mathrm{T}} \ll \epsilon_{\mathrm{T}}$, it dominates already at $\epsilon \sim 1$ leading to a monotonic decrease in $R(\epsilon)$. For $\epsilon_{\mathrm{T}} \ll \gamma_{\phi}^{3} g_{\mathrm{T}} \ll 1$ the temperature dependence of resistance remains qualitatively the same as in Fig. 3. However, the position of the maximum is determined now by $\gamma_{\phi}$ which makes it sensitive to a weak magnetic field. Such a field reduces $\tau_{\phi}$ resulting in the appearance of positive magnetoresistance and a shift of the maximum in $R(\epsilon)$ to lower temperatures. This is in a qualitative agreement with recent experimental measurements [8]. Note that the importance of superconducting fluctuations, e.g. for the magnetoresistance at low $T$, is well known [15]. However, neither the temperature dependence of $R$ near $T_{\mathrm{c}}$ nor the novel MT mechanism were considered before.

In conclusion, we have demonstrated that the resistance of the effectively granular system, characterized by inequalities (11), may have a pronounced maximum as $T$ approaches $T_{\mathrm{c}}$. This maximum is due to the competition between the fluctuation suppression of the one-electron tunneling between grains with the enhancement of transport due to the coherent charge transfer of the fluctuation Cooper pairs. Only at the very edge of the transition the decay in the resistance becomes similar to the monotonic one in the amorphous system. Such a distinctive feature together with the possible sensitivity to weak magnetic fields can serve as a benchmark of the effective granularity of a disordered system.

We thank Allen Goldman and Igor Aleiner for useful discussions of the results. We are grateful to National Center of Theoretical Sciences of R.O.C. for warm hospitality at the final stage of the work. The work was supported by the contract No. DE-AC02-06CH11357 of the U.S. DoE Office of Science. I.V.L. acknowledges support of the EPSRC (EP/D031109) and of the NATO collaborative grant. A.A.V. acknowledges support of the MIUR under the project PRIN 2006 and of the grant RFBR-0702-12058.

[1] D.B. Haviland, Y. Liu, and A.M. Goldman, Phys. Rev. Lett. 62, 2180 (1989).

[2] A. Goldman and N. Markovic, Phys. Today 51, No.11, 39 (1998).

[3] I. S. Beloborodov, A. V. Lopatin, V. M. Vinokur, K. B. Efetov, Rev. Mod. Phys. 79, 469 (2007).

[4] I. S. Beloborodov, K. B. Efetov, A. V. Lopatin and V. M. Vinokur, Phys. Rev. Lett. 91, 246801 (2003).

[5] C. Biagini, T. Caneva, V. Tognetti and A. A. Varlamov, Phys. Rev. B72, 041102(R) (2005).

[6] Ya. M. Blanter, V. M. Vinokur and L. I. Glazman, Phys. Rev. B73, 165322 (2006).

[7] T. I. Baturina et al, Phys. Rev. Lett. 99, 257003 (2007).

[8] K. H. Sarwa, B. Tan, Kevin A. Parendo and A. M. Goldman, arXiv:0704.0765

[9] If the intragrain electron motion is ballistic (a clean superconductor), then the correlation length $\xi_{\mathrm{g}}$ should be substituted by $\xi_{\mathrm{g}}^{\mathrm{b}}=v_{\mathrm{F}} / \Delta$ where $v_{\mathrm{F}}$ is the Fermi velocity in the grain.

[10] A weak disorder in the grain size or shape and in the tunneling amplitude would be irrelevant.

[11] A. A. Varlamov and V. V. Dorin, Zh. Eksp. Teor. Fiz. 84, 1868 (1983).

[12] A. I. Larkin and A. A. Varlamov, Theory of Fluctuations in Superconductors, OUP, Oxford, (2005).

[13] Other contributions of these type vary in different placements of the tunneling amplitudes - circled crosses - on each fermionic loop. A full account of all the AL diagrams is necessary only for an irrelevant numerical coefficient. The same is valid for the MT diagrams.

[14] Here we have expressed $\gamma_{\phi}=\left(T_{\mathrm{c}} \tau_{\phi}\right)^{-1}$ via the standard expressions for $\tau_{\phi}$ in the $3 D$ [16] and $0 D$ [17] cases.

[15] I. S. Beloborodov and K. B. Efetov, Phys. Rev. Lett. 82, 3332 (1999); I. S. Beloborodov, K. B. Efetov and A. I. Larkin, Phys. Rev. B61, 9145 (2000); B. S. Skrzynski, I. S. Beloborodov and K. B. Efetov, Phys. Rev. B65, 094516 (2002).

[16] B. L. Altshuler, A. G. Aronov and D. E. Khmelnitsky, J. Phys. C, 15, 7367 (1982).

[17] U. Sivan, Y. Imry and A. G. Aronov, Europhys. Lett. 28, 115 (1994). 